Research Article

\title{
The Magnetic Bead Computing Model of the 0-1 Integer Programming Problem Based on DNA Cycle Hybridization
}

\author{
Rujie Xu, ${ }^{1}$ Zhixiang Yin $\left(D,{ }^{2}\right.$ Zhen Tang, ${ }^{1}$ Jing Yang, ${ }^{1}$ Jianzhong Cui, ${ }^{1,3}$ and Xiyuan Wang ${ }^{1}$ \\ ${ }^{1}$ School of Mathematics and Big Data, AnHui University of Science \& Technology, Huainan 232001, Anhui, China \\ ${ }^{2}$ School of Mathematics, Physics and Statistics, Shanghai University of Engineering Science, Shanghai 201620, China \\ ${ }^{3}$ School of Electronic and Information Engineering, AnHui University of Science \& Technology, Huainan 232001, Anhui, China
}

Correspondence should be addressed to Zhixiang Yin; zxyin66@163.com

Received 13 October 2020; Revised 4 November 2020; Accepted 4 February 2021; Published 16 February 2021

Academic Editor: Fazal Mahomed

Copyright ( 2021 Rujie Xu et al. This is an open access article distributed under the Creative Commons Attribution License, which permits unrestricted use, distribution, and reproduction in any medium, provided the original work is properly cited.

\begin{abstract}
Magnetic beads and magnetic Raman technology substrates have good magnetic response ability and surface-enhanced Raman technology (SERS) activity. Therefore, magnetic beads exhibit high sensitivity in SERS detection. In this paper, DNA cycle hybridization and magnetic bead models are combined to solve 0-1 integer programming problems. First, the model maps the variables to DNA strands with hairpin structures and weights them by the number of hairpin DNA strands. This result can be displayed by the specific binding of streptavidin and biotin. Second, the constraint condition of the 0-1 integer programming problem can be accomplished by detecting the signal intensity of the biological barcode to find the optimal solution. Finally, this model can be used to solve the general 0-1 integer programming problem and has more extensive applications than the previous DNA computing model.
\end{abstract}

\section{Introduction}

With the development of science and technology, traditional computing has been unable to meet people's requirements when dealing with massive data and information processing, and people have started to explore new fields of computing. Since Adleman proposed the use of DNA computing to solve the directed Hamilton path in 1994, DNA computing has received increasing attention from researchers [1]. In 2000, Head et al. proposed a new method of computing by using DNA plasmids and reported the NP-complete problem concerning the cardinality of the largest independent subset of the vertex set of the computing graph [2]. In 2011, Zhang et al. designed a DNA word set based on minimum free energy [3]. In 2017, Yin and Cui reported the integer programming problem based on the plasmid DNA computing model [4]. In 2018, Ramanamurthy introduced the basic structure of DNA and DNA processing tools [5]. In 2019, Tang established a dynamic NAND computing model using DNA origami [6]. In the same year, Yang et al. used DNA origami and hybridization chain reaction to solve a new computational model for solving the knapsack problem [7].
DNA cycle hybridization chain reaction is a process of alternating hybridization of two DNA molecules with different hairpin structures induced under the induction of a trigger strand. This process is spontaneous and does not require the involvement of enzymes. With the development of science and technology, DNA cycle hybridization chain reaction has been applied to many fields, such as biosensing, biomedicine, proteins, and others. In 2004, Dirks first proposed the concept and indicated that DNA can be used as an amplified transducer for biosensing applications [8]. In 2016, Guo proposed a new chemical immunoassay method for signal amplification that can detect multiple tumor biomarkers simultaneously [9]. In the same year, Yang designed an Aptamer-Binding Directed DNA Origami Pattern for logic gates [10]. In 2018, Li et al. proposed a method for label-free lighting of fluorescent sensors using hybrid chain reaction and DNA triple-strand assembly [11]. In 2018, Xiao designed multiple chemiluminescence imaging and used it for sensitive screening and detection of protein biomarkers through the use of DNA microarray and hybridization chain reaction amplification integration induced by adjacent binding [12]. 
0-1 integer programming is a special type of integer programming problem, and its variable values are only 0 and 1. It is widely used in a variety of problems, such as line design, backpack problems, and dispatch problems [13-17]. In 2006, Yin et al. designed a molecular beacon model for solving integer linear programming problems [13]. In 2010, Huang used the advantage of DNA tiles to build a molecular computing system to solve the $0-1$ programming problem [14]. In 2017, Li devised a new DNA computing method to solve the 0-1 programming problem. The method is based on a self-assembled nanoparticle probe, which reduces the cost of the model and improves the sensitivity and accuracy of detection [17].

In this paper, based on DNA cycle hybridization chain reaction, a magnetic bead model is constructed to solve the general 0-1 integer programming problem. This magnetic bead model maps variables into the hairpin structure DNA strands and maps weights according to the number of hairpin structure DNA strands. This structure can be displayed according to the specific combination of streptavidin and biotin, and SERS detection has been used [18-20]. The general structure of this paper is as follows. First, the basic principle of the DNA cycle hybridization chain reaction and the general form of the $0-1$ integer programming problem are given. Second, the magnetic bead model is constructed, and the algorithm steps of the model and a concrete example are given. Finally, this paper uses Visual DSD software to simulate and analyze the optimal solution of the $0-1$ integer programming problem and then provides the conclusion.

\section{DNA Cycle Hybridization and the 0-1 Integer Programming Problem}

2.1. DNA Cycle Hybridization. DNA cycle hybridization refers to the process in which DNA molecules with complementary base sequences form hydrogen bonds between base pairs to achieve a stable structure. DNA cycle hybridization induces two different types of hairpin structure DNA by using a trigger strand, making the two hairpin structures open alternately and then forming a doublestranded DNA product with a gap. The process of obtaining the DNA product does not require the involvement of enzymes. The reaction principle of DNA cycle hybridization is shown in Figure 1. Two different types of hairpin structures, $H_{1}$ and $H_{2}$, coexist stably in solution without any reaction. $H_{1}$ consists of four parts: $5^{\prime}-l-m-n-m^{*}-3^{\prime}$ ( $m$ and $m^{*}$ base pairs are complementary). It is called the stem of the hairpin. Region $n$ is called the loop of the hairpin structure $H_{1}$, and $l$ is the single-stranded sticky end extended from the stem of $H_{1}$. Similarly, $H_{2}$ consists of four parts: $5^{\prime}-n^{*}-m-l^{*}-m^{*}-3^{\prime}$ ( $m$ and $m^{*}$ base pairs are complementary). It is called the stem of the hairpin, and $n^{*}$ is the single-stranded sticky end extended from the stem of $\mathrm{H}_{2}$. At the same time, $l-l^{*}, m-m^{*}$, and $n-n^{*}$ satisfy the principle of complementary base pairing. The trigger strand $T$ is a single strand of DNA composed of two parts $\left(5^{\prime}-m^{*}-l^{*}-3^{\prime}\right)$. When the trigger strand $T$ is added to the solution, the trigger strand $T$ will have base complementary pairing with the sticky end of $l-m$ of the hairpin structure of $H_{1}$. The resulting hairpin structure $H_{1}$ is opened, exposing the gap $n-m^{*}$. This exposed gap region $n-m^{*}$ happens to have complementary base pairing with $n^{*}-m$ from the $\mathrm{H}_{2}$ region. Opening the structure of $\mathrm{H}_{2}$ to expose the area $l^{*}-m^{*}$, the exposed area $l^{*}-m^{*}$ from $H_{2}$ will continue to pair with the next base complement of $H_{1}$. Opening the structure of $H_{1}$ to expose the area $n-m^{*}$, this exposed gap region will continue to have complementary base pairing with $n^{*}-m$ from the $\mathrm{H}_{2}$ region. Opening the structure of $H_{2}, H_{1}$ and $H_{2}$ are turned on alternately in turn until the sum in the solution is used up. A double-stranded DNA product that hybridizes $H_{1}$ and alternately $H_{2}$ with a gap is generated.

2.2. Integer Programming Problem. 0-1 integer programming is a special type of integer programming problem, and its variable values are only 0 and 1 . The general form of $0-1$ programming is given as follows:

$$
\begin{gathered}
\quad \max (\min ) z=c_{1} x_{1}+c_{2} x_{2}+\cdots+c_{n} x_{n} \\
\left\{\begin{array}{l}
a_{11} x_{1}+a_{12} x_{2}+\cdots+a_{1 n} x_{n} \leq(=, \geq) b_{1} \\
a_{21} x_{1}+a_{22} x_{2}+\cdots+a_{2 n} x_{n} \leq(=, \geq) b_{2} \\
\ldots \ldots \ldots \ldots \ldots \ldots \ldots \\
a_{m 1} x_{1}+a_{m 2} x_{2}+\cdots+a_{m n} x_{n} \leq(=, \geq) b_{m} \\
x_{1}, x_{2}, \ldots, x_{n}=0 \text { or } 1 \\
a_{i j} \text { and } c_{i} \text { are any integer } \\
i=1,2, \ldots, m ; j=1,2, \ldots, n .
\end{array}\right.
\end{gathered}
$$

The most commonly used method for solving the $0-1$ integer programming problem is the exhaustive method (also known as the forced search method), which traverses the entire search space, but this method is time- and laborconsuming.

In this paper, the general $0-1$ integer programming problem is solved based on the DNA cyclc hybridization chain reaction, which is a generalization of the assignment problem.

The algorithm steps of the general 0-1 integer programming problem are discussed as follows:

Step 1. All possible solutions to the problem with variables equal to 0 or 1 are given

Step 2. According to the Raman signal intensity, the nonfeasible solutions in the first constraint condition are eliminated, and the feasible solutions are retained

Step 3. Repeat step 2 with the reserved feasible solutions; then, all nonfeasible solutions can be eliminated, and all feasible solutions of the problem can be obtained

Step 4. The corresponding objective function values of each feasible solution are compared to obtain the optimal solution of the objective function. 


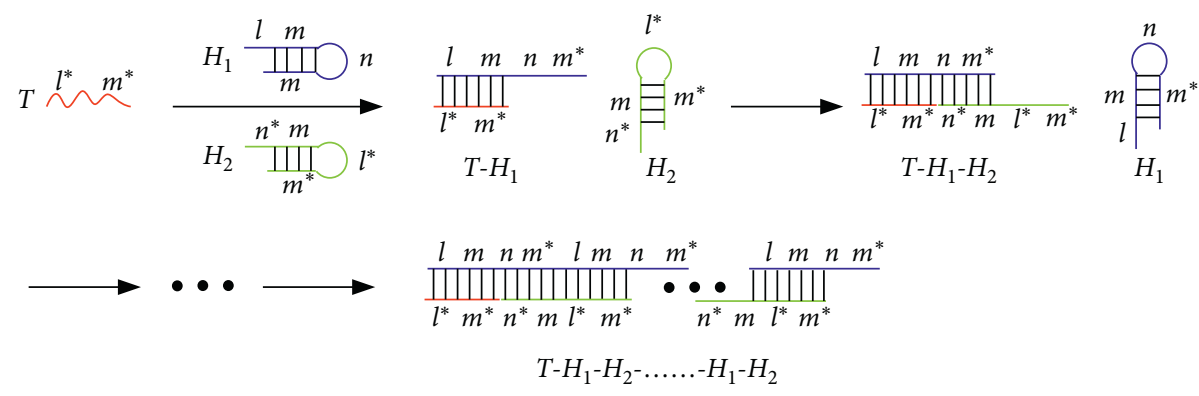

FIgURE 1: The basic principle of DNA cycle hybridization.

\section{Magnetic Bead Model of the 0-1 Integer Programming Problem Based on DNA Cycle Hybridization}

3.1. Building a Magnetic Bead Computing Model. In this paper, we study a new magnetic bead computing model for $0-1$ integer programming problems. The magnetic bead computing model effectively utilizes the specific binding effect of streptavidin and biotin through DNA cycle hybridization technology and Raman technology to detect the signal released by the biological barcode. Table 1 shows the coding sequences of the three DNA molecules. Figure 2 shows the DNA cycle hybridization process.

As shown in Figure 2, at the optimal experimental conditions of $37^{\circ} \mathrm{C}$ and a $\mathrm{pH}$ of 7.4 , the concentration of the captured stranded DNA was $1.0 \times 10^{-7} \mathrm{M}$ [21]. The single strand of capture DNA was fixed on the magnetic bead by means of the amide bond between - $\mathrm{COOH}$ modified by the magnetic bead and - NH2 on the DNA strand. The single strand of capture DNA fixed on the magnetic bead was complementarily paired with the base at the sticky end of the strand of the hairpin DNA1 strand, thus opening the hairpin structure DNA1 strand. When the hairpin structure DNA1 is opened, it is complementary with the sticky end base of the hairpin structure DNA2, thus opening the hairpin structure of DNA2. After the hairpin structure DNA2 is opened, it continues to be complementary to the sticky end of the hairpin structure of DNA1. In this way, the hairpin structure DNA1 strand and DNA2 strand cycle hybridizes successively, forming "magnetic bead-capture DNA-DNA1DNA2-DNA1-...-DNA2-DNA1-DNA2," a special doublestranded DNA molecule. Until DNA1 and DNA2 are consumed in the solution, the sticky end of DNA1 and the sticky end of DNA2 are both modified by biotin and bind to the strepavidin-modified nanobiotic barcode specifically to achieve signal release.

In summary, for the 0 - 1 integer programming problem with $n$ variables $\left(x_{1}, x_{2}, \ldots, x_{n}\right)$ and $m$ constraint equations, the specific algorithm of the general 0-1 integer programming computing model is as follows:

Step 1. First, for $n$ variables in each constraint condition, $n$ magnetic beads with capture DNA were designed (magnetic beads with different radii represent different variables). Second, two types of hairpin structure DNA strands were designed, which were named DNA1 and DNA2. The capture DNA fixed on the magnetic bead can open the hairpin structure DNA1. A gap appeared after the hairpin structure DNA1 was opened, which could be further opened to design the hairpin structure DNA2. In this way, DNA1, DNA2, DNA1, DNA2... cyclically cross each other in turn. Until DNA1 and DNA2 in the solution are consumed (the specific coding sequence design of DNA1 and DNA2 is shown in Table 1 ), when $x_{i}=1$, the sticky ends of the DNA1 strand and the sticky ends of the DNA2 strand were modified with biotin; when $x_{i}=0$, the sticky ends of the DNA1 strand and the sticky ends of the DNA2 strand did not need to be modified with biotin, as shown in Figure 3.

Step 2. First, a proper number of biological barcodes were placed in the data pool and mixed evenly. Second, a set of test tubes was prepared for each constraint condition, and each set of test tubes had $2^{n}$ test tubes (where $n$ represents the number of variables in the constraint condition). Finally, equal amounts of the solution were placed in the desired tubes.

Step 3. For the first constraint, according to the number of possible solutions $k$, take out the test tubes according to step 2 and group them and place a magnetic bead with capture DNA in each test tube of each group. After that, put equal amounts of DNA1 and DNA2 into the solution according to the weight coefficients of the variables in the constraint condition. That is, the total amounts of DNA1 and DNA2 are the same as the weight coefficients of the variables. At the same time, according to the characteristics of DNA cycle hybridization, we alternately put DNA1 and DNA2 into the solution every time and put the DNA1 strand first.

Step 4. When the biological barcode in the solution is combined with the sticky ends of the biotin of DNA1 and DNA2, the cycle hybridization signal will be amplified, and the feasibility solution will be judged by the intensity of amplification of the cycle hybridization signal. Here, it is stated that, when there is no biotin at the sticky ends of DNA1 and DNA2, the signal intensity is 0 , when 1 biological barcode in the solution binds to 1 biotin, the signal intensity is 1 , and so on, and when biological barcodes bind to biotin in the solution, the signal intensity is a, where a represents the coefficient in front of each variable, namely, the weight. 
TABLE 1: The coding sequences of DNA molecules.

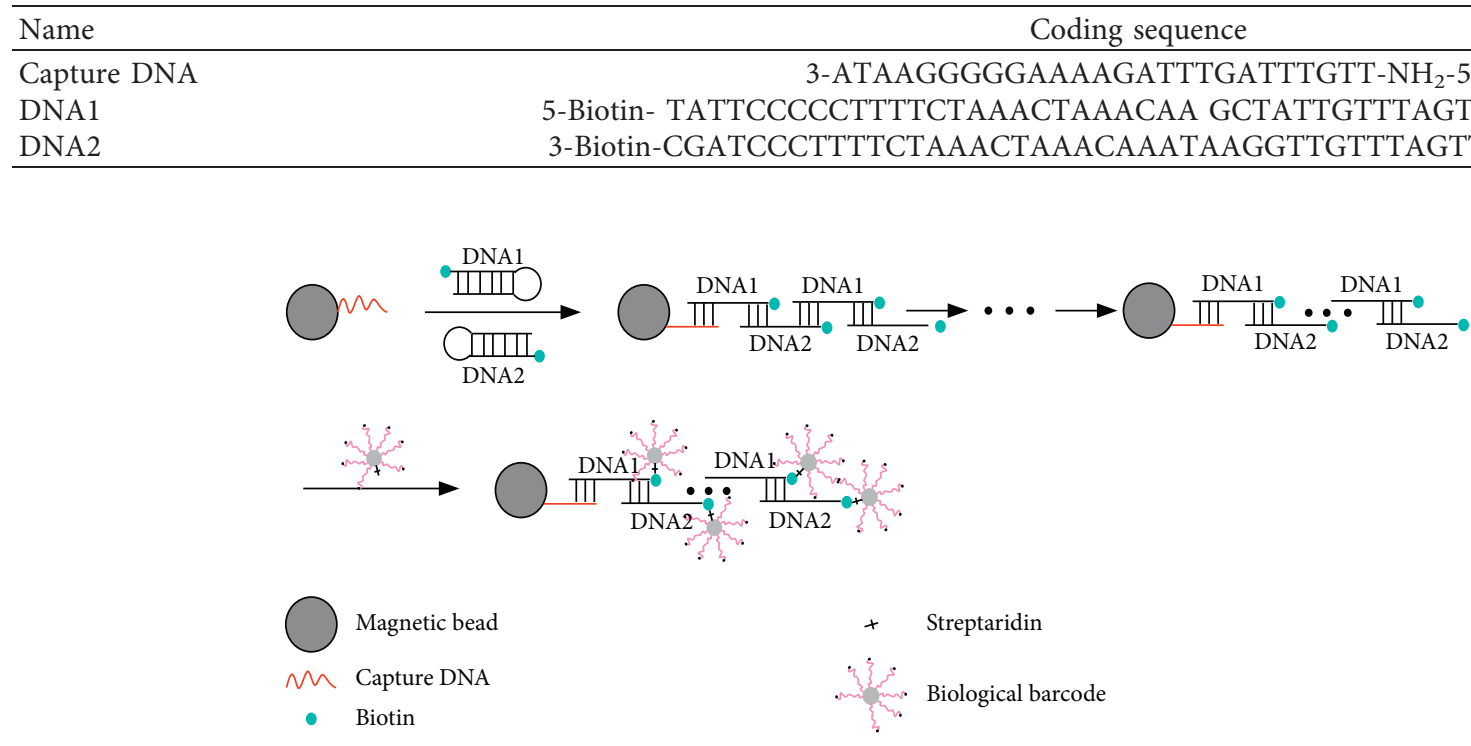

FIGURE 2: The process of DNA cycle hybridization.

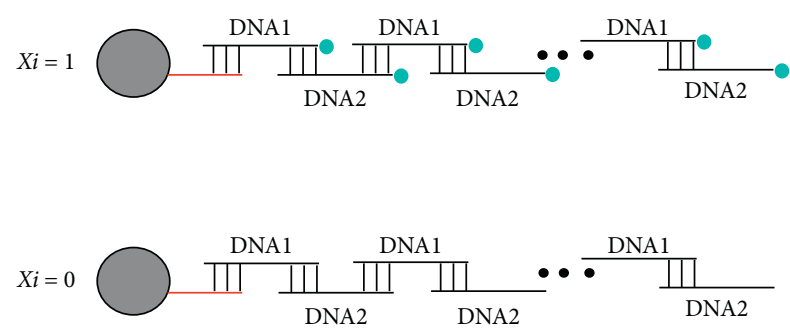

FIgURE 3: The structure diagram of variables $x_{i}=1$ and. $x_{i}=0$.

Step 5. Find a feasible solution satisfying the first constraint condition by detecting the number of biological barcodes.

Step 6. Repeat steps 4-6 above for the feasible solution obtained from the previous constraint condition, and we can obtain the feasible solution that satisfies all of the constraints.

Step 7. Calculate each feasible solution corresponding to the objective function value and, finally, judge the optimal integer programming solution.

3.2. Example Analysis. A general 0-1 integer programming problem is discussed in detail as follows:

$$
\begin{gathered}
\min w=4 x_{1}+3 x_{2}+5 x_{3} \\
\left\{\begin{array}{l}
3 x_{1}+2 x_{2}+4 x_{3} \geq 5 \\
2 x_{1}+3 x_{2} \leq 3 \\
x_{2}+2 x_{3} \leq 2 \\
x_{1}, x_{2}, x_{3}=0 \text { or } 1
\end{array}\right.
\end{gathered}
$$

Step 1. For the variables in each constraint condition, magnetic beads with capture DNA were designed. In this problem, 3, 2, and 2 magnetic beads with capture DNA were designed for the three constraints, with magnetic bead radii of $2 \mathrm{~nm}, 4 \mathrm{~nm}$, and $6 \mathrm{~nm}$, respectively. Then, two types of hairpin structure DNA strands were designed, known as DNA1 and DNA2. When the value of variable $x_{i}$ is 1 , the sticky ends of the DNA1 and DNA2 strands are modified with biotin. When the value of the variable $x_{i}$ is 0 , the sticky ends of the DNA1 and DNA2 strands do not need to be modified with biotin.

Step 2. An appropriate amount of the biological barcode was placed in the solution and mixed evenly. Three sets of test tubes were prepared, and the number of each set of test tubes was 8,4, and 4 . The correct amount and equal amount of solution were placed into the test tubes.

Step 3. All possible solutions of the objective function variables are denoted as $1(0,0,0), 2(0,0,1), 3(0,1,0)$, $4(0,1,1), 5(1,0,0), 6(1,0,1), 7(1,1,0)$, and $8(1,1,1)$. For the first constraint condition, prepare 8 sets of test tubes, which are labeled $1,2,3,4,5,6,7$, and 8, corresponding to the 8 possible solutions of the previous step. There are three test tubes in each set of test tubes, each of which is put into a magnetic bead with captured DNA, which are recorded as $x_{1}, x_{2}, x_{3}$, respectively, and the radii of the magnetic beads are $2 \mathrm{~nm}, 4 \mathrm{~nm}$, and $6 \mathrm{~nm}$, Put DNA1 and DNA2 into the respective test tubes according to the $x_{1}, x_{2}, x_{3}$ coefficients in the constraint condition. Step 4. The specific process is shown in Figure 4.

Step 5. The signal intensities in the 8 test tubes are 0,4 , $2,6,3,7,5$, and 9 . The feasible solutions that satisfy the first constraint condition are $4(0,1,1), 6(1,0,1)$, $7(1,1,0)$, and $8(1,1,1)$.

Step 6. Because the second constraint does not involve $x_{3}$, we only need to consider $x_{1}$ and $x_{2}$. For the feasible solutions obtained in step 6 , the 4 th, 6 th, 7 th, and 8 th groups 


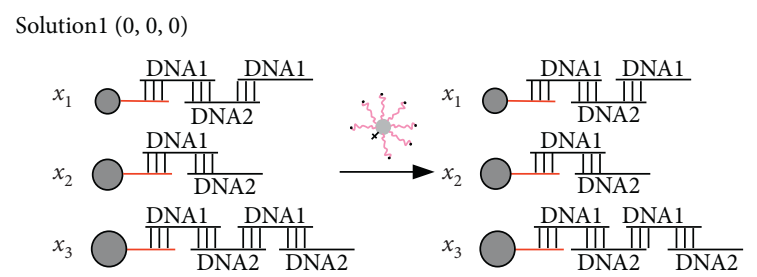

Solution3 $(0,1,0)$

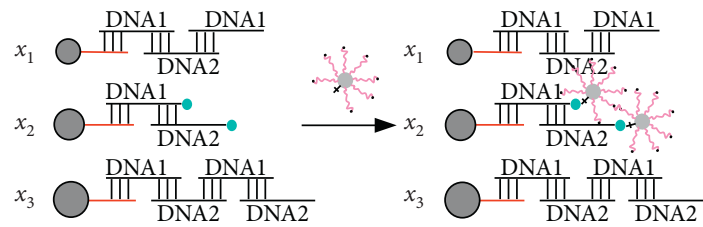

Solution5 $(1,0,0)$
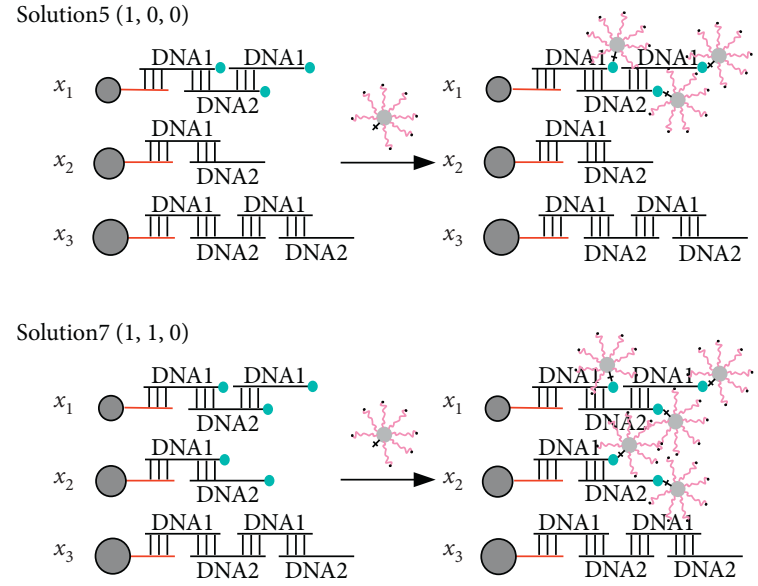

Solution2 $(0,0,1)$
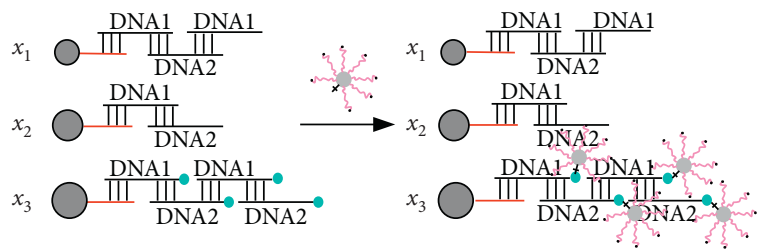

Solution $4(0,1,1)$

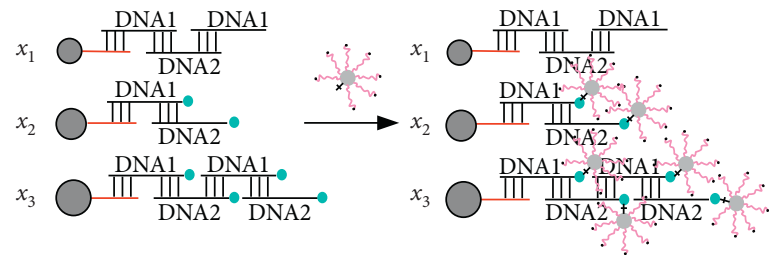

Solution6 $(1,0,1)$
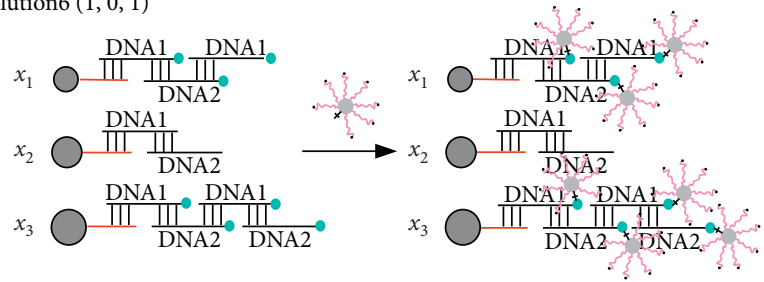

Solution8 $(1,1,1)$

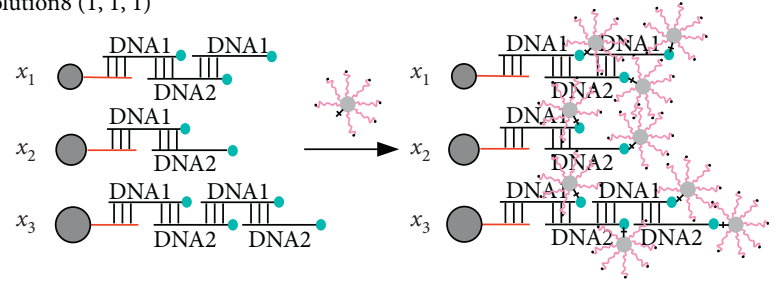

Figure 4: The structure diagram of 8 solutions.

of solutions, the values are $4(0,1), 6(1,0), 7(1,1)$, and $8(1,1)$. Among them, the 7 th and 8 th solutions have the same values, and only the 7 th solution $(1,1)$ is considered here. Continue to steps 4 and 5, as shown in Figure 5.

The signal intensities of these three groups of test tubes, 4,6 , and 7 , are 3,2 , and 5 , respectively. Only group 4 $(0,1)$ and $6(1,0)$ test tubes meet the second constraint. Thus, the feasible solutions satisfying the first two constraints are $4(0,1,1)$ and $6(1,0,1)$.

Because the third constraint condition does not involve variable $x_{1}$, we only need to consider the values $x_{2}$ and $x_{3}$, and the values are $4(1,1)$ and $6(0,1)$. Continue to steps 4 and 5. The specific process is shown in Figure 6 below.

Step 7. Finally, group 6 of solutions $(1,0,1)$ is a feasible solution that satisfies all constraints. Substituting the feasible solution into the objective function, the minimum objective function of the $0-1$ integer programming problem can be obtained as 9 .

\section{Discussion}

Visual DSD is a simulation software commonly used in DNA computing and hybridization chain reaction. This paper uses Visual DSD software to simulate and analyze the optimal solution of the $0-1$ integer programming problem. The optimal solution of the example integer programming problem is $\left(x_{1}, x_{2}, x_{3}\right)=(1,0,1)$. For variables $x_{1}=1$, add the hairpin structure DNA1 strand and DNA2 strand, and because the reaction is just started, the concentration of reactants is higher and the reaction speed is faster. The concentration of the hairpin structure DNA1 and DNA2 strands decreases rapidly in a short time and eventually gradually approaches 0 . For $\mathrm{sp} 5$, the intermediate product of the reaction, because the cycle hybridization reaction is carried out step by step, the concentration of the strand first increases and then decreases before finally approaching 0 . The concentration of the final product sp4 gradually increases and finally tends to be stable. The specific reaction process is shown in Figure 7. The simulation results show that the model is feasible and consistent with the expected results.

Previous models, such as the DNA origami base, circular logic gate, and others, cannot solve the weighted integer programming problem, which increases the understanding space virtually. The magnetic bead model proposed in this paper, which can solve the $0-1$ integer programming problem with weight, can solve the general $0-1$ integer programming problem, so it is more widely used. 

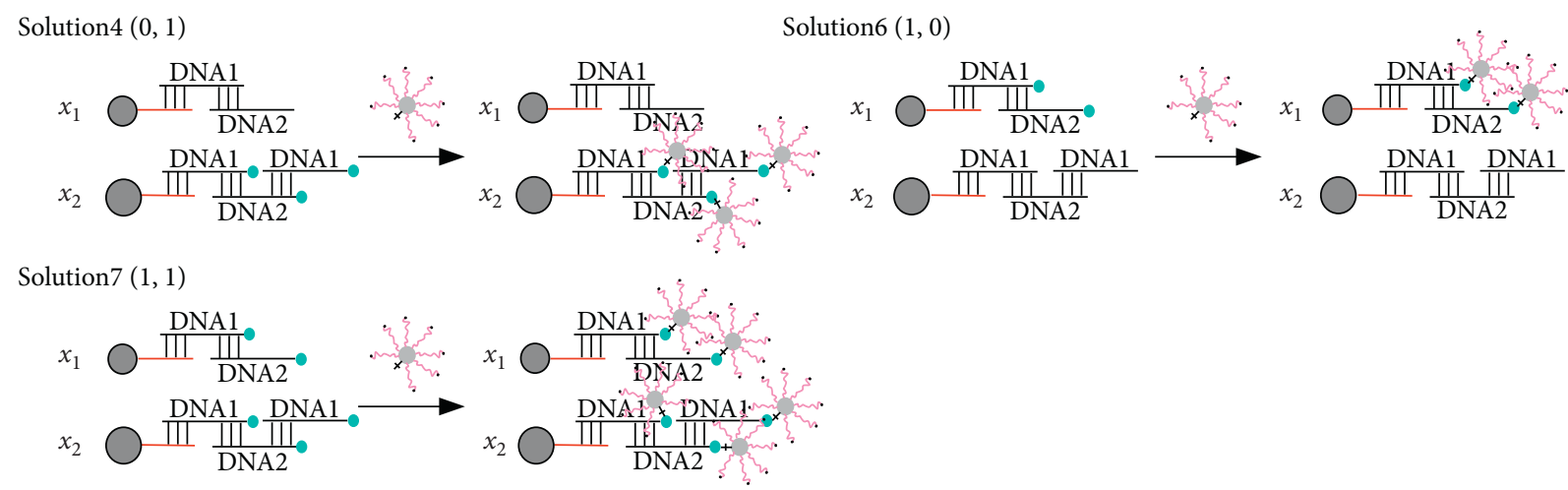

FIGURE 5: Diagram of the solution under the second constraint.

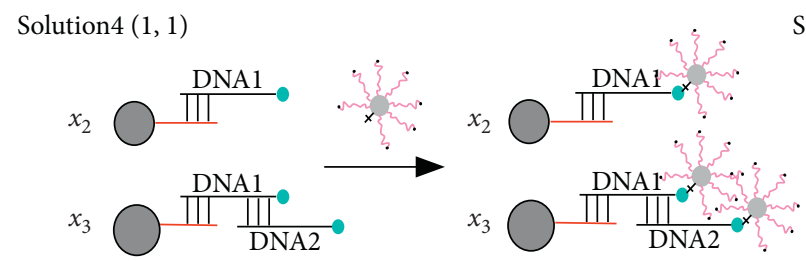

Solution6 $(0,1)$

Figure 6: Diagram of the solution under the third constraint.

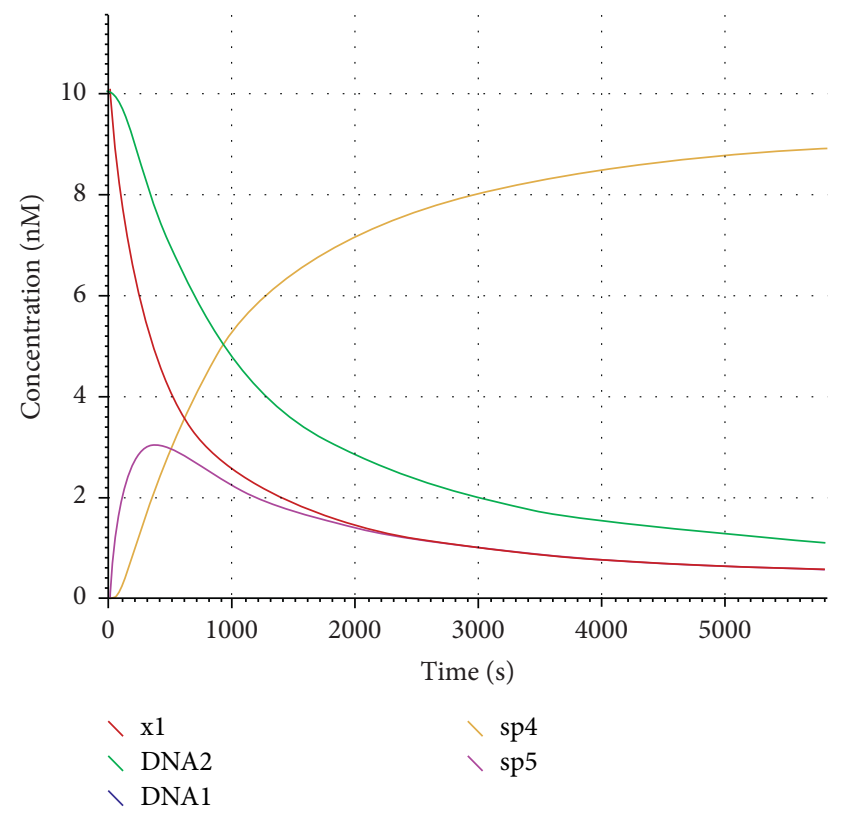

FIGURE 7: Diagram of model simulation.

\section{Conclusion}

In this paper, a magnetic bead model for solving the $0-1$ integer programming problem was established based on the DNA cycle hybridization chain reaction and the specific binding effect of streptavidin and biotin. Compared with the previous DNA computing model, this model has the following advantages. First, there is no requirement for enzymes in the operation process, which can reduce the experimental cost and improve the versatility of the model. Second, the intensity of the signal is used to judge the feasibility of the solution. This can improve the accuracy and practicability of the detection results. Finally, this model can be used to solve the general $0-1$ integer programming problem and has more extensive applications than the previous DNA computing model. However, this method still has some shortcomings, such as a large number of steps and long operation time. Therefore, these aspects still need to be studied further.

\section{Data Availability}

No data were used to support this study.

\section{Conflicts of Interest}

The authors declare that they have no conflicts of interest.

\section{Acknowledgments}

The project was supported by the National Natural Science Foundation of China (nos. 62072296, 61672001, 61702008, and 11971302), Natural Science Foundation of Anhui Province (no. 1808085 MF193), Foreign Visit and Study Project of Outstanding Young Talents in Colleges and Universities (no. gxgwfx2019015), Sub-Project of CST Forward Innovation Project (no. 18163ZT00500901), and Anhui Postdoctoral Fund (no. 2019B331).

\section{References}

[1] L. Adleman, "Molecular computation of solutions to combinatorial problems," Science, vol. 266, no. 5187, pp. 1021-1024, 1994.

[2] T. Head, G. Rozenberg, R. S. Bladergroen, C. K. D. Breek, P. H. M. Lommerse, and H. P. Spaink, "Computing with DNA 
by operating on plasmids," Biosystems, vol. 57, no. 2, pp. 87-93, 2000.

[3] Q. Zhang, B. Wang, X. Wei et al., "DNA word set design based on minimum free energy," IEEE Transactions on Nanobioence, vol. 9, no. 4, pp. 273-277, 2011.

[4] Z. Yin, J. Cui, and J. Yang, "Integer programming problem based on plasmid DNA computing model," Chinese Journal of Electronics, vol. 26, no. 6, pp. 1284-1288, 2017.

[5] S. V. Ramanamurthy, K. Hyndhavi, and B. Sruthi Sai Nirmala, "DNA computing-the future of computing," Journal of Innovation in Computer Sceince Engineering, vol. 8, no. 1, pp. 18-22, 2018.

[6] Z. Tang, Z.-X. Yin, X. Sun, J.-Z. Cui, J. Yang, and R.-S. Wang, "Dynamically NAND gate system on DNA origami template," Computers in Biology and Medicine, vol. 109, pp. 112-120, 2019.

[7] J. Yang, Z. Yin, Z. Tang, K. Huang, J. Cui, and X. Yang, "Search computing model for the knapsack problem based on DNA origami," Materials Express, vol. 9, no. 6, pp. 553-562, 2019.

[8] R. M. Dirks and N. A. Pierce, "From the Cover: triggered amplification by hybridization chain reaction," Proceedings of the National Academy of Sciences, vol. 101, no. 43, pp. 15275-15278, 2004.

[9] J. Guo, J. Wang, J. Zhao, Z. Guo, and Y. Zhang, "Ultrasensitive multiplexed immunoassay for tumor biomarkers based on DNA hybridization chain reaction amplifying signal," ACS Applied Materials \& Interfaces, vol. 8, no. 11, pp. 6898-6904, 2016.

[10] J. Yang, S. Jiang, X. Liu, L. Pan, and C. Zhang, "Aptamerbinding directed DNA origami pattern for logic gates," ACS Applied Materials \& Interfaces, vol. 8, no. 49, pp. 3405434060, 2016.

[11] Z. Li, L. Tingting, S. Ruidi et al., "A label-free light-up fluorescent sensing platform based upon hybridization chain reaction amplification and DNA triplex assembly," Talanta, vol. 189, pp. 137-142, 2018.

[12] Q. Xiao, J. Wu, P. Dang, and H. Ju, "Multiplexed chemiluminescence imaging assay of protein biomarkers using DNA microarray with proximity binding-induced hybridization chain reaction amplification," Analytica Chimica Acta, vol. 1032, pp. 130-137, 2018.

[13] Z. X. Yin, J. Z. Cui, J. Yang, and X. Yang, BDNA Computing Model of the Integer Linear Programming Problem Based on Molecular Beacon, Springer, Berlin, Germany, 2006.

[14] X. Zheng, J. Yang, C. Zhou, C. Zhang, Q. Zhang, and X. Wei, "Allosteric DNAzyme-based DNA logic circuit: operations and dynamic analysis," Nucleic Acids Research, vol. 47, no. 3, pp. 1097-1109, 2019.

[15] Y. Huang, Z. Cheng, J. Xu, X. Shi, and K. Zhou, "Solving 0-1 planning problem based on self-assembly of DNA tiles," Journal of Computational and Theoretical Nanoscience, vol. 7, no. 5, pp. 826-830, 2010.

[16] J. Yang, C. Zhang, S. Liu, H. Xia, and J. Xu, "A molecular computing model for 0-1 programming problem using DNA nanoparticles," Journal of Computational and Theoretical Nanoscience, vol. 10, no. 10, pp. 2380-2384, 2013.

[17] F. Li, "DNA computation based on self-assembled nanoparticle probes for 0-1 integer programming problem," Science, vol. 268, no. 5210, pp. 542-546, 2017.

[18] A. M. Michaels, M. Nirmal, and L. E. Brus, "Surface enhanced Raman spectroscopy of individual rhodamine 6G molecules on large Ag nanocrystals," Journal of the American Chemical Society, vol. 121, no. 43, pp. 9932-9939, 1999.
[19] H. Zhang, C. Fang, and S. Zhang, "An autonomous biobarcode DNA machine for exponential DNA amplification and its application to the electrochemical determination of adenosine triphosphate," Chemistry-A European Journal, vol. 16, no. 41, pp. 12434-12439, 2010.

[20] B. Wang, Q. Zhang, and X. Wei, "Tabu variable neighborhood search for designing DNA barcodes," IEEE Transactions on NanoBioscience, vol. 19, no. 1, pp. 127-131, 2020.

[21] Y. Lu, G. L. Liu, and L. P. Lee, "High-density silver nanoparticle film with temperature-controllable interparticle spacing for a tunable surface enhanced Raman scattering substrate," Nano Letters, vol. 5, no. 1, pp. 5-9, 2005. 